\title{
Perspective: SARS-CoV-2, COVID-19 and Haematologists
}

\author{
Robert Peter Gale \\ Centre for Haematology Research, Department of Immunology and Inflammation, Imperial College London, \\ London, UK
}

Severe acute respiratory syndrome-coronavirus-2 (SARS-CoV-2) and the resultant disease, coronavirus infectious disease-2019 (COVID-19) pose a challenge to haematologists. People we typically treat are high-risk for opportunistic infections because of bone marrow failure and immune suppression from drugs targeting bone marrow function or both. Here, I make a distinction between lymphoid and myeloid cancers, especially B-cell cancer. Lymphoid cancers such as lymphomas and chronic lymphocytic leukaemia are associated with profound immune suppression because of direct involvement of the immune system in the cancer. In the context of SARSCoV-2 infection, the immune system, adaptive and innate, are important defenses against virus infections. This is also true of recipients of haematopoietic cell transplants who receive immune suppressive drugs before and after transplantation and in whom the donor-derived immune system is defective to varying degrees. Add to these complications such as acute and chronic graft-versus-host disease and you have an ultra-high-risk population.

The situation differs in myeloid cancers where granulocyte and macrophage production are defective because of the disease and or its therapy. Here, the primary susceptibility is to bacterial, fungal, and parasitic not virus infections. For example, persons with acute myeloid leukaemia receiving bone marrow-damaging but not immune suppressive drugs have a normal skin graft rejec-

karger@karger.com

(c) 2020 S. Karger AG, Basel

www.karger.com/aha

Karger! tion time. However, in the context of COVID-19, many deaths result not directly from SARS-CoV-2 but from opportunistic infections and/or an uncontrolled host immune response similar or identical to cytokine release syndrome (CRS).

What do we know about SARS-CoV-2-infection and COVID-19 in persons with haematological cancers? My Chinese colleagues and I studied several aspects of this in Wuhan, epicentre of the SARS-CoV-2 pandemic. Complete typescripts are available at https://www.nature.com/ leu/ with open access.

First, we conducted a cohort study of 128 hospitalized subjects with haematological cancers at two centres in Wuhan, China. Thirteen (10\%) developed COVID-19. As controls we studied 226 health care providers $16(7 \%)$ of whom developed COVID-19 and 11 of whom were hospitalized. Case rates for COVID-19 in hospitalized subjects with haematological cancers and health care providers were similar. Co-variates were compared with 115 subjects with haematological cancers without COVID-19 and with the 11 hospitalized health care providers with COVID-19. There were no significant differences in baseline co-variates between subjects with haematological cancers developing or not developing COVID-19. However, the 13 subjects with haematological cancers had more severe COVID-19 and more deaths compared with the hospitalized health care providers with COVID-19. 
Case fatality rates were $62 \%$ and none. We concluded hospitalized persons with haematological cancers have a similar case rate of COVID-19 compared with normal health care providers but have more severe disease and a higher case fatality rate. Because we were unable to identify specific risk factors for COVID-19 in hospitalized persons with haematological cancers, we suggested increased surveillance and possible protective isolation [1].

In another study, we used a questionnaire to study 530 subjects with chronic myeloid leukaemia (CML) in Hubei Province. Five developed confirmed $(n=4)$ or probable COVID-19 $(n=1)$. Prevalence of COVID-19 in our subjects, $0.9 \%$, was 9 -fold higher than the $0.1 \%$ reported in normals but lower than the $10 \%$ reported in hospitalized persons with other haematological cancers (cited above) and normal health care providers, $7 \%$. Co-variates associated with an increased risk of developing COVID-19 amongst persons with CML were exposure to someone infected with SARS-CoV-2, no complete haematological remission and/or co-morbidities. There was also an unexplained increased risk of COVID-19 in subjects with advanced-phase CML at diagnosis even after achieving a complete cytogenetic response or major molecular response at the time they developed COVID-19. One of 21 subjects receiving third-generation tyrosine kinase inhibitor (TKI)-developed COVID-19 versus 3 out of 346 subjects receiving imatinib versus none of 162 subjects receiving second-generation TKIs. Because the choice of TKI was not random, we could not exclude the role other co-variates operated. For example, persons with poorrisk leukaemia may have been more likely to receive a third-generation TKI; and it is always important to emphasize that a correlation or association does not necessarily imply cause-and-effect. Other co-variates, such as interval from diagnosis and therapy duration, were not significantly associated with an increased risk of developing COVID-19. We concluded persons with these risk factors may benefit from increased surveillance of SARS$\mathrm{CoV}-2$ infection and possible protective isolation [2].

Our third study was a meta-analysis to determine safety and efficacy of corticosteroids in persons with COVID-19 and other severe coronavirus infections including SARS-CoV and MERS-CoV. Efficacy endpoints studied included death, hospitalization duration, rates of intensive care unit (ICU) admission and use of mechanical ventilation, and a composite endpoint of death, ICU admission or mechanical ventilation. We included 11 reports involving 5,247 subjects. Corticosteroid use was associated with delayed virus clearing with no significant reduction in the risk of death. Hospitalization duration was prolonged and use of mechanical ventilation increased. The composite endpoint worsened. We concluded that corticosteroid use in subjects with SARS-CoV-2, SARS-CoV, and MERS-CoV infections delays virus clearing and does not convincingly improve survival, reduce hospitalization duration or ICU rate admission, and/or use of mechanical ventilation and there were several adverse effects. Because there were mostly observational studies and selection and publication biases, our conclusions, especially regarding SARS-CoV-2, need confirmation. In the interim we suggest caution using corticosteroids in persons with COVID-19 [3].

There are many more important and unanswered questions for haematologists, especially physicians involved in haematopoietic cell transplants. Recently, we published suggested guidelines to help centres manage transplant patients and donors [4]. Guidelines for infection control from the European Hematology Association (EHA) are also available at https://www.nature.com/leu/. Most of these guidelines, as those of several organizations such as the European Bone Marrow Transplant Group (EBMT) and American Society for Transplantation and Cellular Therapy (ASTCT), are not evidenced-based. Although they make sensible suggestions, many of the recommendations may prove wrong if ever tested in a controlled randomized trial. Because this is unlikely to happen, the recommenders are safe, at least for now. Caveat emptor.

Little is known about the impact of the SARS-CoV-2 pandemic on other cell therapies such as chimeric antigen receptor (CAR)-T-cell therapy. Here, there may be good and bad news. Because the procedure typically involves autologous $\mathrm{T}$ cells (allogeneic CAR-T cells are occasionally used) the issue of possible infection of an allogeneic donor is gone. However, there may still be transportation restrictions if the product processing centre is remote from the recipient etc. The bad news is that CAR-T-cell recipients are already at high risk of CRS, which, unfortunately, is a common feature of severe COVID-19. Think of double-hit lymphomas. Although there are reasonably convincing data of safety and efficacy on interleukin-6 antagonists in CRS related to CAR-T-cell therapy [5] there are only anecdotal reports of this approach in COVID-19 pneumonia [6]. US FDA has approved a phase-3 placebo-controlled trial of tocilizumab in COVID-19 pneumonia (https://clinicaltrials.gov/ct2/show/ NCT04320615), and US National Institutes of Allergy and Infectious Disease (NIAID) is about to start a phase-3 study of baricitinib and/or remdesivir in persons with COVID-19 pneumonia, which was suggested by an arti- 
ficial intelligence programme as a potentially promising approach [7]. One of two trials of remdesivir reported a slight shortening of recovery interval; clearly not a home run [8].

Detailed discussions of safety and efficacy of other potential intervention such as mesenchymal stromal cells and natural killer cells, are beyond the scope of this perspective. Preliminary results of phase- 2 trails are being reported, but data are mostly uninterpretable regarding efficacy with appropriate controls. Elsewhere, my colleagues and I review the possible role of mesenchymal stromal cells in COVID-19-related acute respiratory distress syndrome [9]. Others are studying placenta or umbilical cord-derived natural killer cells (for example, https://www.europeanpharmaceuticalreview.com/ news/116794/us-researchers-to-study-stem-cell-therapy-in-covid-19-patients/). If cell therapy-based approaches prove safe and effective, operationalizing this will involve haematologists, especially those in cell therapy and/or haematopoietic cell transplantation.

A final word about how the SARS-CoV-2 pandemic has interrupted the orderly progression of scientific and medical research. Hopefully, today most haematologists practice what is termed evidence-based medicine. However, it is useful to recall that this term was first used in the biomedical literature only 30 years ago by Gordon Guyatt. The process was initially termed scientific medicine. The response of our colleagues was less than enthusiastic because of the implication prior medical decisions were unscientific. Undaunted, Guyatt tried evidencebased medicine; it stuck. Who today would practice voodoo-based rather than evidence-based medicine?

However, this is a bit of The Emperor's New Clothes. It is worth recalling that evidence-based medicine is not new. Hippocrates, Aesculapius, Maimonides, Avicenna and others made recommendations based on careful, critical observations. However, the first randomized clinical trial is attributed to the British naval surgeon James Lind. In 1753, he published his account of the comparative treatment of sailors with scurvy: "their cases as similar as I could have them." He divided 12 sick sailors into six pairs, giving each pair a different dietary supplement: cider, diluted sulfuric acid, vinegar, sea water, two oranges, and a lemon or a purgative. He noted: "The most sudden and visible good effects were perceived from the use of the oranges and lemons." So much for power calculations. (One can only wonder what an Institutional Review Board would make of the sulfuric acid cohort).

Progress toward understanding whether therapies were safe and effective was slow but steady. Hundred and

SARS-CoV-2, COVID-19 and

Haematologists forty-five years later, Johannes Fibiger, a Danish physician, studied 484 persons with diphtheria giving them a serum treatment or not based on what day they were admitted to a Copenhagen hospital. Fibiger argued for the need "to eliminate completely the play of chance and the influence of subjective judgment," indicating a clear understanding of the hazards of uncontrolled comparisons. Many scientists, physicians, epidemiologists and statisticians, too numerous to mention, realized the need for scientific rigor in the evaluation of the safety and efficacy of medical interventions.

Along came the SARS-CoV-2 pandemic and COVID-19. Suddenly, we have disruption of 267 years of progress. People with no scientific education or credentials are promoting drugs of unknown safety and efficacy based on flimsy, sometimes discredited reports from uncontrolled clinical trials. Chloroquine, hydroxychloroquine and azithromycin come to mind. Internal disinfectants and UV-light quickly followed. Some, but fortunately not most, physicians have joined this stampede arguing there is "nothing to lose." Unfortunately, there is much to lose. Firstly, there are safety concerns about these and other drugs. For example, one might make the same argument about corticosteroids in COVID-19. However, as I discussed above, corticosteroids are not only ineffective in COVID-19, they are associated with adverse events and outcomes. More important is the loss of scientific rigour and discipline. We cannot afford to let our supposedly new-found evidence-based medicine be hijacked by politicians, charlatans and the like. Fortunately, most haematologists are acting sensibly and with careful, controlled studies of drugs to treat COVID-19. I am optimistic that we will get through this crisis with our scientific principles intact.

\section{Acknowledgement}

I thank Prof. Qiubai Li and his colleagues from Wuhan, Prof. Qian Jiang and her colleagues from Hebei Provence and Beijing, Yang Liang and his colleagues from Guangzhou, Prof. He Huang and his colleagues from Hangzhou and Dr. Hamdan Hamdan and his colleagues from Riyadh and Houston for their collaboration in the studies cited. R.P.G. acknowledges support from the National Institute of Health Research (NIHR) Biomedical Research Centre funding scheme.

\section{Disclosure Statement}

The author declares that he has no conflicts of interest to disclose. 


\section{References}

1 He W, Chen L, Chen L, Yuang G, Fang Y, Chen W, et al. COVID-19 in persons with haematological cancers. Leukemia. Forthcoming 2020. DOI: $10.1038 /$ S41375-0200836-7.

2 Li W, Wang D, Guo J, Yuan G, Yang Z, Gale $\mathrm{RP}$, et al. COVID-19 in persons with chronic myeloid leukaemia. Leukemia. Forthcoming 2020. https://www.nature.com/leu/.

3 Li H, Chen C, Hu F, Wang J, Zhao Q, Gale RP, et al. Evidence against use of corticosteroids SARS-CoV-2, SARS-CoV and MERS-CoV infections. Leukemia. Forthcoming 2020. https://www.nature.com/leu/.
4 Xiao H, Luo Y, Shi J, Jin A, Mohty M, Nagler A, et al. Recommendations for hematopoietic stem cell transplantation during the $\mathrm{CO}$ VID-19 outbreak: experience and perspective from China. Bone Marrow Transplant. Forthcoming 2020. https://www.nature.com/bmt/.

5 Kennedy LB, Salama AK. A review of cancer immunotherapy toxicity. CA Cancer J Clin. 2020 Mar; 70(2):86-104.

6 Luo P, Liu Y, Qiu L, Liu X, Liu D, Li J. Tocilizumab treatment in COVID-19: A single center experience. J Med Virol. 2020 Apr 6. https://doi.org/10.1002/jmv.25801.

7 Richardson P, Griffin I, Tucker C, Smith D, Oechsle O, Phelan A, et al. Baricitinib as potential treatment for 2019-nCoV acute respiratory disease. Lancet. 2020 Feb;395(10223): e30-1.
8 Grein J, Ohmagari N, Shin D, Diaz G, Asperges E, Castagna A, et al. Compassionate use of remdevisir for patients with severe $\mathrm{Co}$ vid-19. N Engl J Med. 2020 Apr 10;NEJMoa2007016. https://doi.org/10.1056/NEJMoa2007016.

9 Hamden H, Hashmi SK, Lazarus HM, Gale RP, Qu W, Fakih RE. Possible role of mesenchymal stromal cells in coronavirus infectious disease-19-related severe acute respiratory syndrome. Bone Marrow Transplant. Forthcoming 2020. https://www.nature. $\mathrm{com} / \mathrm{bmt} /$. 